\title{
Arizona Sonora Binational Cases: Five Years of Surveillance
}

\author{
Mariana G. Casal ${ }^{\star 1,}$, , Raquel M. Bravo-Clouzet ${ }^{1,2}$, Irene Ruberto ${ }^{1,3}$, Teresa Jue ${ }^{1,3}$, \\ Robert Guerrero', 2 and Kenneth Komatsu ${ }^{1,3}$
}

'Office of Border Health, Arizona Department of Health Services, Tucson, AZ, USA; ${ }^{2}$ Office of Border Health, Tucson, AZ, USA;

${ }^{3}$ Bureau of Epidemiology and Disease Control, Phoenix, AZ, USA

\section{Objective}

To describe 5 years of binational infectious disease surveillance using the binational variable in the medical electronic surveillance system in Arizona

\section{Introduction}

Infectious diseases can spread across borders. (1) The Arizona Department of Health Services (ADHS) collects information on binational infectious disease cases and shares it with Mexico. Infectious disease investigation in Arizona is enhanced by using an electronic surveillance platform known as the Medical Electronic Disease Surveillance Intelligence System (MEDSIS), and in 2010 a specific variable for binational cases with Mexico was added to the platform. ADHS also maintains a binational case definition in the state reportable communicable morbidities manual. Arizona partners with the US Centers for Disease Control and Prevention (CDC)'s Division of Global Migration and Quarantine (DGMQ), US Mexico Unit (USMU), in a monthly binational case reporting project, and shares information with the Ministry of Health of the State of Sonora, Mexico, (SON MOH) to reinforce ongoing communication, to establish baseline disease patterns, and to help detect binational outbreaks. In 2007, the Ministry of Health of the State of Sonora began to use the MEDSIS system for real-time secure case notification, and secure file sharing, using the Arizona's Health Services Portal and secure e-mail accounts for confidential communication between both states.

\section{Methods}

From 2011 to 2015, the ADHS Binational Border Infectious Disease Surveillance (BIDS) program maintained a database to collect information on binational cases with Mexico, and coordinated regularly with $\mathrm{SON} \mathrm{MOH}$ to investigate and respond to binational cases and outbreaks. In addition, a SAS program was created to search for possible binational cases not designated as binational using variables such as an address in Mexico and Mexican citizenship. The ADHS BIDS program investigated all suspected binational cases with Mexico and classified them as binational according to the case definition established by the Council of State and Territorial Epidemiologists (CSTE). The ADHS BIDS program also shared binational cases from Mexico with CDC DGMQ USMU through an Epi-X Forum and with SON MOH through MEDSIS or secure e-mail.

\section{Results}

Between 2011 and 2015, the ADHS BIDS program investigated 2,158 possible binational cases with Mexico. From those, $70.44 \%$ $(n=1520)$ were classified as binational with Mexico according with the CSTE case definition. The majority of cases were classified as binational because of travel to Mexico ( $\mathrm{n}=1089,71.6 \%$ ), with $59 \%$ traveling to Sonora $(\mathrm{n}=641)$. The majority of cases during those 5 years were enteric diseases $(n=1086,71.4 \%)$, followed by vector borne diseases $(n=131,8.6 \%)$. Most of the binational cases reported had symptom onset between June and August, following the seasonality of both southbound travel and enteric diseases. Regular communication with Sonora facilitated detection of an average of three binational outbreaks per year. All confirmed binational cases were reported to CDC DGMQ USMU and SON MOH.

\section{Conclusions}

Continuous sharing of infectious disease surveillance information between both states is essential in understanding the magnitude and types of reportable diseases in the Arizona/Sonora border region. Proper use of the binational MEDSIS variable enables a quicker identification of binational status, allowing for the prompt investigation of possible binational cases and detection of binational outbreaks. Binational outbreaks led to collaborative Arizona/Sonora investigations on several occasions, strengthening our relationship, coordination, collaborations, and understanding of the surveillance system used by SON MOH. Real-time exchange of information using the same secure surveillance system enables better situational awareness, more timely and accurate binational communication, and binational collaborations.

Arizona Sonora Binational Cases

\begin{tabular}{|c|c|c|}
\hline Year & Binational Cases Investigated & Confirmed Binational Cases \\
\hline 2011 & 272 & 208 \\
\hline 2012 & 235 & 172 \\
\hline 2013 & 423 & 254 \\
\hline 2014 & 560 & 406 \\
\hline 2015 & 668 & 480 \\
\hline
\end{tabular}

\section{Keywords}

binational variable; reportable; infectious diseases; border region

\section{Acknowledgments}

BIDS program at US Mexico Unit San Diego and Arizona Department of Health Services.

\section{References}

1. Barnett ED, Walker PF. Role of immigrants and migrants in emerging infectious diseases. Med Clin North Am. 2008 Nov; 92(6):1447-58, xi-xii. doi: 10.1016/j.mcna.2008.07.001

\section{*Mariana G. Casal}

E-mail: mariana.casal@azdhs.gov 